\title{
Modeling charge collection efficiency degradation in partially depleted GaAs photodiodes using the 1- and 2- carrier Hecht equations
}

\author{
E. C. Auden ${ }^{1}$, G. Vizkelethy, D. K. Serkland, D. J. Bossert, B. L. Doyle
}

\begin{abstract}
The Hecht equation can be used to model the nonlinear degradation of charge collection efficiency (CCE) in response to radiation-induced displacement damage in both fully and partially depleted GaAs photodiodes. CCE degradation is measured for laser-generated photocurrent as a function of fluence and bias in $\mathrm{Al}_{0.3} \mathrm{Ga}_{0.7} \mathrm{As} / \mathrm{GaAs} /$ $\mathrm{Al}_{0.25} \mathrm{Ga}_{0.75} \mathrm{As}$ p-i-n photodiodes which have been irradiated with $12 \mathrm{MeV} \mathrm{C}$ and $7.5 \mathrm{MeV}$ Si ions. CCE is observed to degrade more rapidly with fluence in partially depleted photodiodes than in fully depleted photodiodes. When the intrinsic GaAs layer is fully depleted, the 2-carrier Hecht equation describes CCE degradation as photogenerated electrons and holes recombine at defect sites created by radiation damage in the depletion region. If the GaAs layer is partially depleted, CCE degradation is more appropriately modeled as the sum of the 2-carrier Hecht equation applied to electrons and holes generated within the depletion region and the 1-carrier Hecht equation applied to minority carriers that diffuse from the field-free (non-depleted) region into the depletion region. Enhanced CCE degradation is attributed to holes that recombine within the field-free region of the partially depleted intrinsic GaAs layer before they can diffuse into the depletion region.
\end{abstract}

Keywords: charge collection efficiency; gallium arsenide; ion radiation effects; ion accelerators; photodiodes

\section{Introduction}

The electrical performance of irradiated semiconductor devices exhibits long-term degradation as energetic particles introduce displacement damage in the semiconductor lattice. Lattice defects with energy levels near the middle of the bandgap can efficiently trap both electrons and holes, leading to reduced gain in bipolar junction transistors (BJTs), increased leakage current in diodes, and hot pixels in CCDs [1]. Radiation-induced displacement damage degrades charge collection efficiency (CCE) in photodiodes, where CCE is the ratio of measured photocurrent $P C$ to the initial photocurrent $P C_{0}$ generated in an undamaged photodiode [2]. Leakage current increases linearly with fluence while BJT gain degradation is inversely proportional to fluence, but CCE exhibits a nonlinear response to radiation damage.

In 1932, Karl Hecht developed an analytical model for the amount of charge that can be collected at the anode of a crystal with light shining on it [3]. Hecht's equation calculates the ratio of collected charge to photogenerated charge as a function of 1) the drift length of carriers and 2) the distance between the point at which charge is generated and the point at which it is induced. The recombination time $\tau$ associated with a carrier is the average amount of time elapsed between its generation and subsequent recombination. The drift length $\lambda$ is the average distance that a carrier will travel in a material before recombination occurs, and $\lambda$ can be expressed as the product of recombination time and drift velocity, $\lambda=v \tau$. Three physical processes contribute to $\tau$, band-to-band recombination, Shockley-Read-Hall (SRH) recombination at midgap defects, and Auger recombination [4]. Band-to-band recombination is the primary recombination process in undamaged, low-doped GaAs, but $\tau$ is reduced mainly by SRH recombination as displacement damage accumulates.

Hecht's model was originally developed for electrons (the 1-carrier Hecht equation), but it has been extended to incorporate holes and electrons (the 2-carrier Hecht equation) to describe CCE in semiconductor devices. Photogenerated electron / hole pairs ( $\mathrm{e}^{-} / \mathrm{h}$ pairs) drifting through a depletion region contribute to measured photocurrent until they recombine or move out of the depletion region. This process is described by the Gunn theorem [5] from which Hecht's equation can be derived. In photodiodes, as in Hecht's crystal, only a fraction of photogenerated charge can be collected since holes and electrons may recombine before they can be collected. In a modern, pristine photodiode, over $99 \%$ of photogenerated charge can be collected since the drift length for electrons

\footnotetext{
${ }^{1}$ Corresponding author. Tel. +1 505844 0536, fax + 1505844 7775, email eauden@ sandia.gov. Address: Sandia National Laboratories, P.O. Box 5800 MS 0665, Albuquerque, NM, 87185-0665

Submitted for review on October 19th, 2016. This work was sponsored by the Laboratory Directed Research and Development program at Sandia National Laboratories, Laboratories which is a multi-program laboratory managed and operated by Sandia Corporation, a wholly owned subsidiary of Lockheed Martin Corporation, for the U.S. Department of Energy's National Nuclear Security Administration under contract DE-AC04-94AL85000.
} 
(and holes) is on the order of centimeters, while the distance between the points of generation and collection on the order of microns. However, as displacement damage accrues in a photodiode exposed to radiation, electrons and holes will recombine at an increasing number of mid-bandgap defects. The drift length becomes shorter, and CCE is reduced.

Recent work by Vittone et al. demonstrates that Hecht's equation can model CCE degradation in ion-irradiated Si diodes [6] by using the Messenger-Spratt equation $\left(\tau^{-1}=\tau_{0}^{-1}+k \Phi\right)$ [7] to express recombination time as a function of fluence $\Phi$ and a k-factor specific to a device and ion species / energy. In [6], CCE degradation is calculated as the ratio of measured ion-beam induced charge (IBIC) signal to the charge generated by an ion pulse incident upon a Si diode. In this work, an $808 \mathrm{~nm}$ laser is used to generate continuous photocurrent in GaAs p-i-n photodiodes as they are irradiated with heavy ion pulses. CCE degradation is calculated as the ratio of photocurrent measured between ion pulses to the initial photocurrent level measured in a pristine photodiode. Measured CCE degradation is compared to an analytical model that incorporates the Hecht equation. CCE degradation is also simulated for a GaAs p-i-n photodiode with recombination lifetimes between $10 \mathrm{~ns}$ and $0.1 \mathrm{ps}$ using the Silvaco ATLAS device simulation framework [8]. Simulation results are presented with experimental data to validate the analytical model which shows that the 2-carrier Hecht equation can model radiation-induced CCE degradation in fully depleted photodiodes, but a combination of the 1-carrier and 2-carrier Hecht equations is appropriate for partially depleted photodiodes.

\section{Materials and Methods}

\subsection{GaAs photodiode structure}

The GaAs p-i-n photodiodes irradiated in this work were designed and fabricated at Sandia National Laboratories (SNL) resulting in a device very similar to the PIN photodiode in [9]. Fig. 1 shows nominal material thicknesses and doping levels for these photodiodes. The $0.5 \mu \mathrm{m} p$-type $\mathrm{Al}_{0.3} \mathrm{Ga}_{0.7} \mathrm{As}$ layer is doped with $10^{18} \mathrm{~cm}^{-3}$ of $\mathrm{Be}$, and the $0.7 \mu \mathrm{m}$ "intrinsic" layer of GaAs is unintentionally doped n-type at an effective concentration of $10^{16} \mathrm{~cm}^{-3}$. The built-in bias at this junction creates a depletion region that extends $0.42 \mu \mathrm{m}$ into the intrinsic layer from the $p n$ junction. The intrinsic GaAs layer can therefore be subdivided into a $0.42 \mu \mathrm{m}$ depletion region in which the electric field is nonzero and a $0.28 \mu \mathrm{m}$ field-free region. As the magnitude of reverse bias is increased, the depletion region widens and the field-free region becomes narrower. The GaAs layer is fully depleted at an applied bias of approximately $-2.2 \mathrm{~V}$. Below the intrinsic GaAs layer, there is a $0.2 \mu$ m layer of $n$-type $\mathrm{Al}_{0.25} \mathrm{Ga}_{0.75} \mathrm{As}$ doped with $10^{18} \mathrm{~cm}^{-3}$ of $\mathrm{Si}$, a $0.5 \mu \mathrm{m}$ layer of $n$-type $\mathrm{Ga}_{0}$ As doped with $10^{18} \mathrm{~cm}^{-3}$ of $\mathrm{Si}$, and a GaAs substrate several hundred microns thick (not shown in Fig. 1 or the structure simulated in this work). The photodiode described in [9] has a $0.05 \mu \mathrm{m}$ surface layer of GaAs; we have replaced this layer with Au to serve has an electrode in electrical simulations. The lateral dimensions of the experimental photodiodes were $100 \mu \mathrm{m} \times 100 \mu \mathrm{m}$, although the 2-D

\begin{tabular}{|ll|}
\hline $\mathrm{Au}$ & $0.05 \mu \mathrm{m}$ \\
\hline $\mathrm{Al}_{0.3} \mathrm{Ga}_{0.7} \mathrm{As}\left(\mathrm{p}, 10^{18} \mathrm{Be}\right)$ & $0.5 \mu \mathrm{m}$ \\
\hline GaAs $\left(\mathrm{n}, 10^{16} \mathrm{Si}\right)$ & $0.7 \mu \mathrm{m}$ \\
\hline $\mathrm{Al}_{0.25} \mathrm{Ga}_{0.75} \mathrm{As}\left(\mathrm{n}, 10^{18} \mathrm{Si}\right)$ & $0.2 \mu \mathrm{m}$ \\
\hline $\mathrm{GaAs}\left(\mathrm{n}, 10^{18} \mathrm{Si}\right)$ & $0.5 \mu \mathrm{m}$ \\
\hline $\mathrm{Au}$ & $0.05 \mu \mathrm{m}$ \\
\hline
\end{tabular}

Fig. 1: Material stack used for GaAs photodiode simulations.

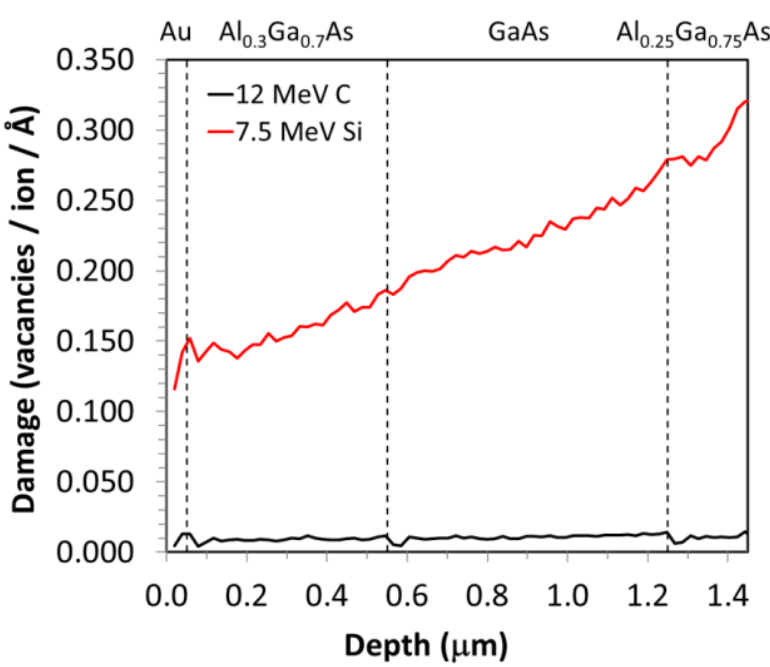

Fig. 2: Simulated damage profiles for $12 \mathrm{MeV} \mathrm{C}$ and $7.5 \mathrm{MeV}$ Si. 
Table I:

Summary of Irradiation Parameters

\begin{tabular}{ccccccc}
\hline Ion & Flux & $\begin{array}{c}\text { Initial pulse } \\
\text { length }\end{array}$ & Initial fluence & $\begin{array}{c}\text { Number } \\
\text { of pulses }\end{array}$ & $\begin{array}{c}\text { Total fluence } \\
\text { Vacancies } \\
/ \mathrm{cm}^{3}\end{array}$ \\
\hline $12 \mathrm{MeV} \mathrm{C}$ & $1.7 \times 10^{13} \mathrm{~cm}^{-2} \mathrm{~s}^{-1}$ & $100 \mu \mathrm{s}$ & $1.7 \times 10^{9} \mathrm{~cm}^{-2}$ & 11 & $3.4 \times 10^{12} \mathrm{~cm}^{-2}$ & $3.4 \times 10^{17}$ \\
$12 \mathrm{MeV} \mathrm{C}$ & $4.0 \times 10^{12} \mathrm{~cm}^{-2} \mathrm{~s}^{-1}$ & $400 \mu \mathrm{s}$ & $1.7 \times 10^{9} \mathrm{~cm}^{-2}$ & 11 & $3.7 \times 10^{12} \mathrm{~cm}^{-2}$ & $3.7 \times 10^{17}$ \\
$7.5 \mathrm{MeV} \mathrm{Si}$ & $6.8 \times 10^{13} \mathrm{~cm}^{-2} \mathrm{~s}^{-1}$ & $10 \mu \mathrm{s}$ & $6.8 \times 10^{8} \mathrm{~cm}^{-2}$ & 18 & $2.7 \times 10^{11} \mathrm{~cm}^{-2}$ & $5.4 \times 10^{17}$ \\
\hline
\end{tabular}

ATLAS simulations of the device described in 2.4 assume that the device is $1.7 \mu \mathrm{m}$ wide (that is, as wide as it is deep).

\subsection{CCE measured in irradiated photodiodes}

GaAs photodiodes were irradiated with $12 \mathrm{MeV}$ C and 7.5 MeV Si ions generated with the SNL 6 MV High Voltage Engineering Corporation (HVEC) tandem Van de Graaff accelerator [10]. Two diodes were irradiated with pulses of $12 \mathrm{MeV} \mathrm{C}$ ions, and one diode was irradiated with pulses of 7.5 MeV Si ions. Successive ion pulse lengths were increased by a factor of $\sim 2$ for the $12 \mathrm{MeV} \mathrm{C}$ ions and a factor of $\sim 1.5$ for the $7.5 \mathrm{MeV}$ Si ions. The fluence delivered by each pulse was approximated from ion beam induced current (IBIC) in the photodiode by software installed at the Tandem accelerator. Each photodiode was irradiated until photocurrent had degraded to $\sim 50 \%$ of the initial photocurrent measured in an undamaged device. After each ion beam pulse, photocurrent was measured while irradiating the photodiode with an $808 \mathrm{~nm}$ laser. The laser power was $10 \mathrm{~mW}$. Fig. 2 shows damage profiles for 12 $\mathrm{MeV} \mathrm{C}$ and $7.5 \mathrm{MeV}$ in a GaAs photodiode structure simulated with the Stopping Range of Ions in Matter (SRIM) code [11]. SRIM calculations of vacancy generation rates assumed a displacement threshold energy of $10 \mathrm{eV}$ for GaAs[12]. Table I summarizes the ion species and energy, flux, initial pulse length, initial fluence, number of pulses, and total fluence for each irradiation as well as the vacancy generation rate calculated with SRIM.

Fig. 3 shows photocurrent vs. bias measured in the $7.5 \mathrm{MeV} \mathrm{Si-irradiated} \mathrm{photodiode.} \mathrm{A} \mathrm{pre-irradiation} \mathrm{IV} \mathrm{curve}$ is shown along with IV curves measured after 6 of the 17 ion pulses. The bandgap of the $p$-type $\mathrm{Al}_{0.3} \mathrm{Ga}_{0.7} \mathrm{As}$ layer is $1.80 \mathrm{eV}$, and the n-type GaAs layer has a bandgap of $1.42 \mathrm{eV} \mathrm{[13].} \mathrm{The} 808 \mathrm{~nm}$ laser produces $1.53 \mathrm{eV}$ photons that pass through the $p$-type $\mathrm{Al}_{0.3} \mathrm{Ga}_{0.7} \mathrm{As}$ layer with no attenuation before creating $\mathrm{e}^{-} / \mathrm{h}$ pairs in the GaAs intrinsic layer. Before the device is irradiated, photocurrent magnitude is independent of applied bias over the range $-4 \mathrm{~V}$ to $+0.5 \mathrm{~V}$. This behavior is consistent with drift and diffusion lengths which are much longer than the GaAs layer thickness of $0.7 \mu \mathrm{m}$; when the GaAs layer is fully depleted, holes and electrons are likely to drift (or drift and diffuse in the case of partial depletion) out of the GaAs layer before they can recombine, so photocurrent is constant over the applied bias range shown in Fig. 1. As fluence (and therefore displacement damage) increases, holes and electrons are more likely to recombine within the GaAs layer before they can drift or diffuse out, so the dependence of photocurrent magnitude on applied bias becomes apparent in Fig. 3. The GaAs photodiodes are designed to have pre-irradiation charge collection efficiency near $100 \%$, so CCE was measured as the as the ratio of post-irradiation photocurrent to

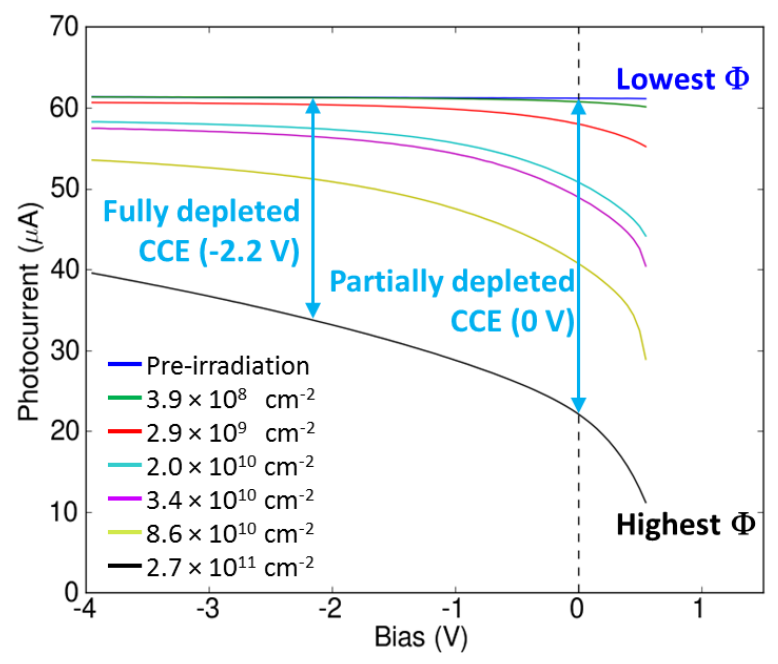

Fig. 3: Photocurrent vs. bias in device irradiated with 7.5 MeV Si.

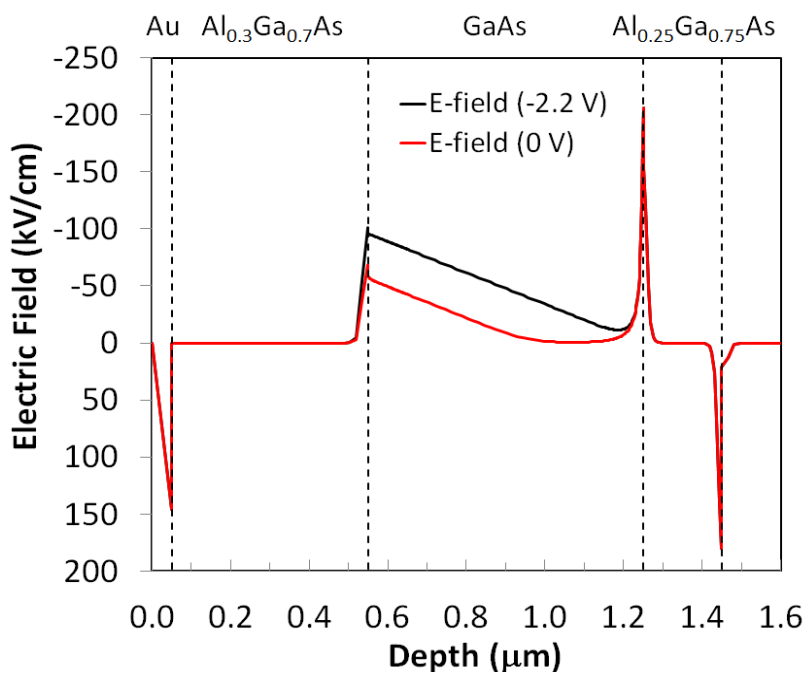

Fig. 4: Electric field vs. depth under the surface for the device in Fig. 1. 


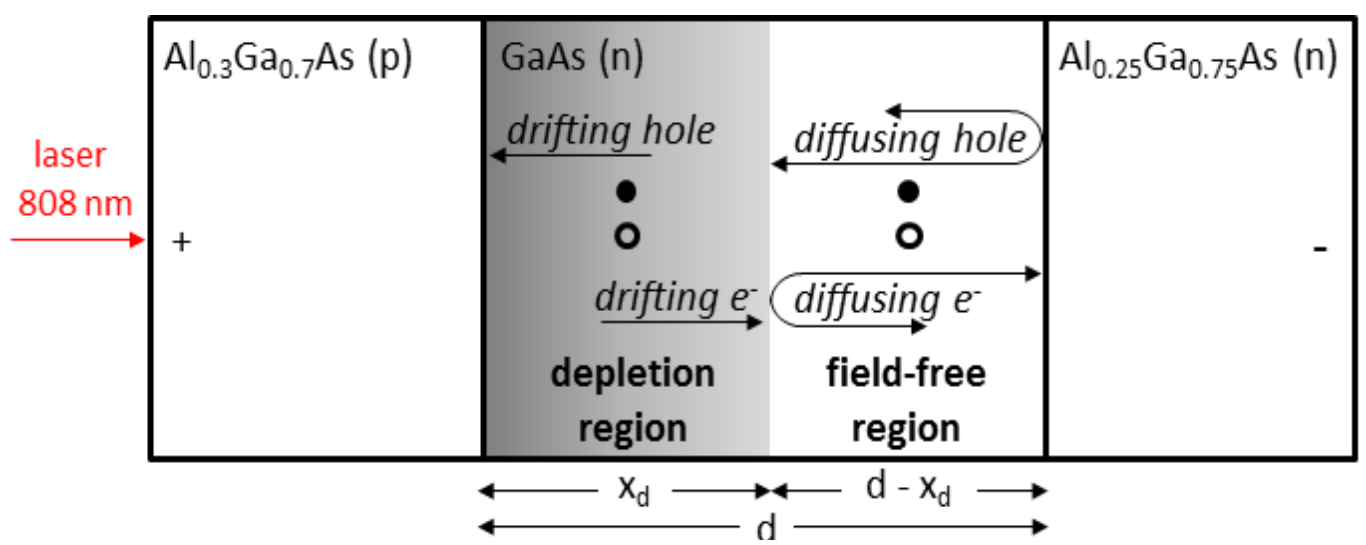

Fig. 5. Cartoon of carrier motion in the depletion region and field-free region of a partially depleted GaAs photodiode.

pre-irradiation photocurrent. CCE decreased with fluence at all bias conditions, but CCE decreased more slowly when the device was reverse-biased such that the intrinsic layer was fully depleted. For example, Fig. 3 shows that photocurrent decreased by $25 \mu \mathrm{A}(42 \%)$ after exposure to $2.7 \times 10^{11} \mathrm{~cm}^{-2}$ ions of $7.5 \mathrm{MeV}$ Si when the photodiode was fully depleted with a bias of $-2.2 \mathrm{~V}$. After exposure to the same fluence, photocurrent decreased by $38 \mu \mathrm{A}$ $(63 \%)$ when the photodiode was partially depleted with a bias of $0 \mathrm{~V}$.

\subsection{CCE calculated with the Hecht equation}

When a DC laser generates photocurrent $P C_{0}$ in the fully depleted intrinsic layer of a p-i-n photodiode, the carriers drift in the electric field and induce photocurrent $P C$ in the measurement circuit as described by the Gunn theorem [5]. Since the DC laser generates a steady-state photocurrent, the CCE ratio $P C / P C_{0}$ can be simplified to $Q / Q_{0}$, where $Q_{0}$ is the amount of charge generated in a given sampling period and $Q$ is the corresponding amount of charge induced in the measurement circuit. The 1-carrier Hecht equation given in (1) describes the charge $Q$ induced when photogenerated holes or electrons drift a distance $x$ from the point-of-generation to the boundary of an electric field [3]. Fig. 4 compares the electric fields present in the GaAs layer when the device is partially depleted at a bias of $0 \mathrm{~V}$ and fully depleted at a bias of $-2.2 \mathrm{~V}$.

$$
\frac{Q}{Q_{0}}=\int_{0}^{x_{d}} \frac{Q_{0} e^{-x / \lambda}}{x_{d}} d x=\frac{\lambda}{x_{d}}\left(1-e^{-x_{d} / \lambda}\right)
$$

In (1), the depletion region width is $x_{d}$, and the average drift distance $\lambda$ (or drift length) for electrons is the product of carrier recombination lifetime $\tau$ and drift velocity $v$. The 2-carrier Hecht equation defines the ratio $Q / Q_{0}$ in terms of the drift lengths of both electrons $\lambda_{e}$ and holes $\lambda_{h}$ :

$$
\frac{Q}{Q_{0}}=\frac{\lambda_{h}}{x_{d}}\left[1-\frac{\lambda_{h}}{x_{d}}\left(1-e^{-x_{d} / \lambda_{h}}\right)\right]+\frac{\lambda_{e}}{x_{d}}\left[1-\frac{\lambda_{e}}{x_{d}}\left(1-e^{-x_{d} / \lambda_{e}}\right)\right]
$$

If all $\mathrm{e}^{\mathrm{e}} / \mathrm{h}$ pairs are generated inside a fully depleted intrinsic layer of width $x_{d}$, the 2-carrier Hecht equation given in (2) expresses CCE as $Q / Q_{0}$. Calculation of CCE with the Hecht equation in (2) assumes that the charge generation profile is uniform across the GaAs layer. CCE can also be calculated using the Gunn theorem and an exponential charge generation profile. Appendix A demonstrates the convergence of the Gunn theorem and the 2-carrier Hecht equation in (2) for these conditions.

If the intrinsic GaAs layer is partially depleted, $\mathrm{e}^{-/ \mathrm{h}}$ pairs are generated in both the depletion region and the fieldfree region, the expression for CCE given in (2) must be adapted since carriers generated within the field-free region are transported through diffusion rather than drift. Fig. 5 shows a cartoon of carrier motion in the intrinsic layer of a partially depleted GaAs photodiode in which $x_{d}$ is the depletion region width and $d$ is the intrinsic layer width. Carriers generated in the field-free region of width $d-x_{d}$ are subject to diffusion rather than drift. Holes can diffuse from the field-free region to the depletion region, but electrons are repelled from diffusing into the depletion region 
by the direction of its electric field, so they do not contribute to CCE. Electrons generated within the field-free region can diffuse into the $n$-type AlGaAs barrier layer, but this is another field-free region. The interface between the $n$-type GaAs layer and the $n$-type AlGaAs barrier layer acts as a mirror to diffusing holes. A fraction of holes generated in the field-free region will recombine before they can diffuse into the depletion region. Holes that reach the depletion region will drift toward the $p$-type $\mathrm{Al}_{0.3} \mathrm{Ga}_{0.7} \mathrm{As}$ layer, and the CCE contribution of these holes is modeled by the 1-carrier Hecht equation given in (1).

When a laser with absorption coefficient $A$ is incident upon the surface of a partially depleted GaAs photodiode, the fraction of charge carriers photogenerated in the depletion region is $f_{d r}$, and the fraction of carriers generated within the field free region is $f_{f r}$. These two quantities can be expressed as

$$
f_{d r}=\left\{\frac{1-e^{-A x_{d}}}{1-e^{-d A}}\right\}
$$

and

$$
f_{f r}=\left\{\frac{e^{-A x_{d}}\left(1-e^{-A\left(d-x_{d}\right)}\right)}{1-e^{-d A}}\right\} .
$$

A fraction of holes generated within the field-free region will survive recombination and diffuse into the depletion region. The 1-carrier Hecht equation does not apply to holes as they are diffusing within the field-free region; it only applies to holes that have survived field-free region recombination to diffuse into the depletion region where they contribute to CCE as they drift. The fraction of surviving holes $h_{f r}$ generated in the field-free region can be approximated as

$$
h_{f r}=e^{-\alpha\left(d-x_{d}\right) / L_{h}}
$$

The hole diffusion length $L_{h}$ is defined in terms of hole lifetime $\tau_{h}$ and hole diffusion coefficient $D_{h}$ as $L_{h} \equiv\left(D_{h} \tau_{h}\right)^{1 / 2}$ [14]. The average fraction of the field-free region through which holes must diffuse to reach the depletion region is $\alpha$, and it is set here to $1 / 2$. In a simplified view of the field-free region, holes generated at the field-free region / depletion region boundary would traverse $0 \%$ of the field-free region to diffuse into the depletion region $(\alpha=0)$ holes generated at the boundary of the field-free region and the $n$-type AlGaAs layer would need to traverse $100 \%$ of the field-free region to diffuse into the depletion region $(\alpha=1)$. We set $\alpha=0.51 / 2$ as an approximation that, on average, holes diffusing into the depletion region must first traverse $50 \%$ of the field-free region. It should be noted that this simplified view ignores the holes that traverse more than $100 \%$ of the field-free region if they are "reflected" off the $n$-type AlGaAs boundary.

CCE can now be expressed for a partially depleted GaAs photodiode, using equations (1) - (4), as the sum of the 2 -carrier Hecht equation (for $\mathrm{e}^{-} / \mathrm{h}$ pairs generated within the depletion region) and the 1 -carrier Hecht equation (for holes that diffuse from the field-free region into the depletion region):

$$
C C E=f_{d r}\left(\frac{\lambda_{h}}{x_{d}}\left(1-\frac{\lambda_{h}}{x_{d}}\left(1-e^{-\frac{x_{d}}{\lambda_{h}}}\right)\right)+\frac{\lambda_{e}}{x_{d}}\left(1-\frac{\lambda_{e}}{x_{d}}\left(1-e^{-\frac{x_{d}}{\lambda_{e}}}\right)\right)\right)+f_{f r}\left(h_{f r}\right)\left(\frac{\lambda_{h}}{x_{d}}\left(1-e^{-x_{d} / \lambda_{h}}\right)\right) .
$$

The average drift distances $\lambda_{e(h)}$ and the hole diffusion length $L_{h}$ can be expressed in terms of carrier lifetimes $\tau_{e(h)}$, so these two terms hold the key to calculating CCE as displacement damage accumulates. The MessengerSpratt equation [7] can be invoked to express average drift distances in terms of $\mathrm{k}$-factor and fluence as $\lambda_{e(h)}=v_{e(h)} / k \Phi$ if $\left(\tau_{h 0, e}\right)^{-1}<<k \Phi$. At low electric fields, electron drift velocity $v_{e}$ is the position-dependent product of electric field strength and electron mobility. Similarly, hole drift velocity $v_{h}$ is the product of electric field strength and hole mobility. As electric field strength increases, the drift velocity of electrons saturates at $\sim 7.7 \times 10^{6} \mathrm{~cm} / \mathrm{s}$ in GaAs [15]. ATLAS simulations indicate that hole drift velocity saturates at the same value at a bias of $-4 \mathrm{~V}$ for the devices used in this work.

For the calculations below, the expression for $v_{e(h)}$ has been simplified to the product of average electric field strength and low-field carrier mobility since electric field strength varies linearly with position on either side of a pn-junction in a diode [14]. The Messenger-Spratt equation can also be used to express hole diffusion length in terms of $k \Phi$ instead of recombination lifetime $\tau_{h}$ as $L_{h}=\left(D_{h} / k \Phi\right)^{1 / 2}$. Therefore, the equation for CCE in a partially depleted photodiode given in (5) can be expressed in terms of $k \Phi$ instead of $L_{h}$ and $\lambda_{e(h)}$ :

$$
C C E=f_{d r}\left(\frac{v_{h}}{x_{d} k \Phi}\left(1-\frac{v_{h}}{x_{d} k \Phi}\left(1-e^{-\frac{x_{d} k \Phi}{v_{h}}}\right)\right)+\frac{v_{e}}{x_{d} k \Phi}\left(1-\frac{v_{e}}{x_{d} k \Phi}\left(1-e^{-\frac{x_{d} k \Phi}{v_{e}}}\right)\right)\right)
$$




$$
+f_{f r}\left[e^{-\sqrt{\frac{\alpha^{2}\left(d-x_{d}\right)^{2} k \Phi}{D_{h}}}}\right]\left(\frac{v_{h}}{x_{d} k \Phi}\left(1-e^{-x_{d} k \Phi / v_{h}}\right)\right)
$$

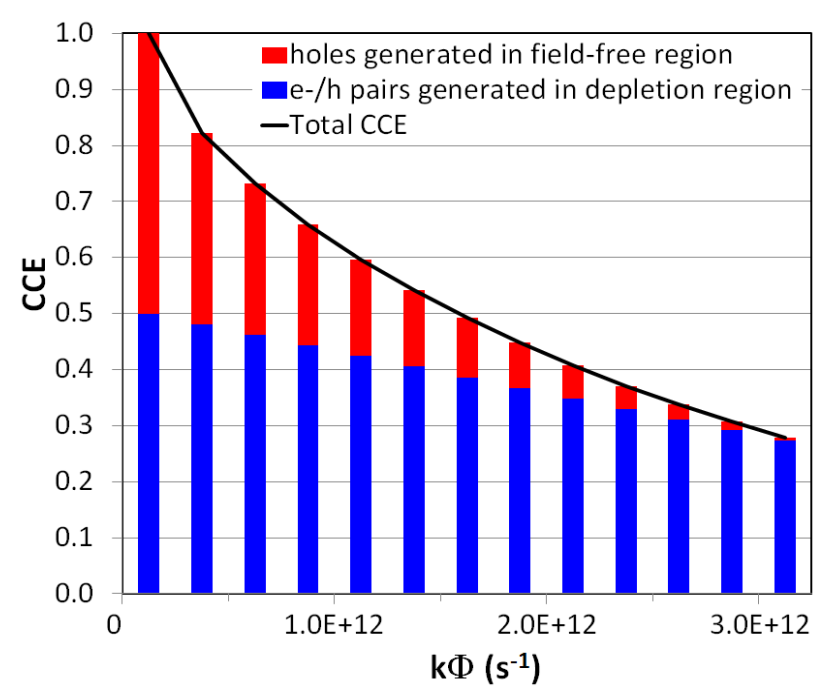

Fig. 6. Relative contribution to CCE in a partially depleted photodiode made by holes generated in the field-free region and e-/h pairs generated in the depletion region.

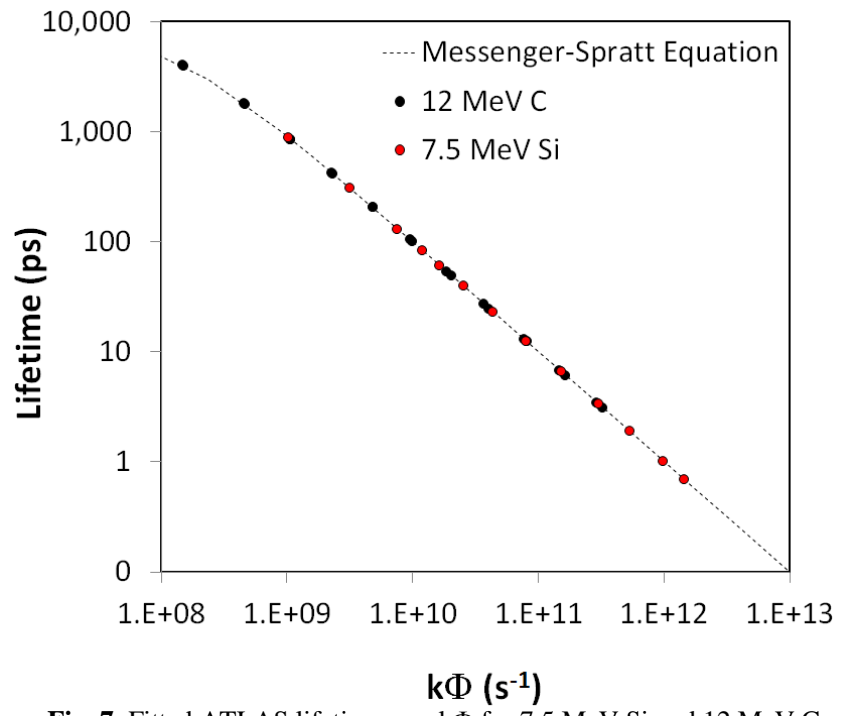

Fig. 7. Fitted ATLAS lifetimes vs. $\mathrm{k} \Phi$ for $7.5 \mathrm{MeV}$ Si and $12 \mathrm{MeV} \mathrm{C}$.

A first order Taylor series expansion of (6) shows that CCE has a linear dependence on $k \Phi$ in the depletion region. In the field-free region, $\mathrm{CCE}$ has both a linear and square root dependence on $k \Phi$ :

$$
C C E(\phi)=C C E_{d r}+C C E_{f r} \approx f_{d r}\left[1-\frac{1}{6} \frac{x_{d} k \phi}{v_{h}}-\frac{1}{6} \frac{x_{d} k \phi}{v_{e}}\right]+f_{f r}\left[1-\sqrt{\frac{\alpha^{2}\left(d-x_{d}\right)^{2} k \phi}{D_{h}}}\right]\left[1-\frac{1}{2} \frac{x_{d} k \phi}{v_{h}}\right] .
$$

Fig. 6 uses the Taylor series expansion given in (7) to show the relative contribution to CCE of holes generated in the field-free region (red dataset) and e-/h pairs generated in the depletion region for a photodiode in which half of the intrinsic layer is depleted. At low fluences, CCE decreases more rapidly with fluence in partially depleted photodiodes because holes generated within the field-free region recombine at defect sites before they can diffuse into the depletion region. Once $k \Phi$ reaches an approximate value of $D_{h} / \alpha^{2}\left(d-x_{d}\right)^{2}$, only carriers generated within the depletion region will contribute to CCE. For example, if half of the intrinsic layer is depleted $\left(x_{d}=1 / 2 d\right)$ and the laser generates $50 \%$ of carriers in the depleted region and $50 \%$ in the field-free region, once $k \Phi \approx D_{h} / \alpha^{2}\left(d-x_{d}\right)^{2} \approx 3 \times 10^{12}$ then no more holes generated in the field-free region will survive to diffuse into the depletion region.

\subsection{CCE simulated with ATLAS}

Computer simulations of the bias- and damage-dependence of CCE degradation were performed using the Silvaco ATLAS TCAD program [8]. Laser-generated photocurrent was simulated through the inclusion of a C language module that calculates the photogeneration rate $G$ in units of $\mathrm{e}^{-} / \mathrm{h}$ pairs $/ \mathrm{cm}^{3} / \mathrm{s}$ as a function of depth $x$ :

$$
G=\frac{P_{0}}{\pi r^{2}} \frac{\lambda_{l}}{h c} \frac{1}{q} A e^{-A x}
$$

where $P_{0}$ is the laser power, $r$ is the laser spot radius, $\lambda_{l}$ is laser wavelength, $h$ is Planck's constant, $c$ is the speed of light, $q$ is the electronic charge, and $A$ is the laser absorption coefficient. The photogeneration rate for an $808 \mathrm{~nm}$ laser was calculated with an absorption coefficient of $1.2 \times 10^{4} \mathrm{~cm}^{-1}$ [16]. The Blaze III/V semiconductor module was enabled to tailor charge transport behavior in the GaAs and AlGaAs layers present in the material stack shown in Fig. 1. Note that the highly conductive GaAs substrate is replaced with an Au metallization in this stack.

The displacement damage resulting from increasing ion bombardment was simulated by decreasing the fixed Shockley Read Hall carrier lifetimes for electrons and holes from an initial value of $10 \mathrm{~ns}$ to $0.1 \mathrm{ps}$. Carrier lifetimes $\tau_{e(h)}$ are related to defect density $N_{t}$, thermal velocity $v_{t h}$, and electron and hole capture-cross sections $\sigma_{e(h)}$ through 


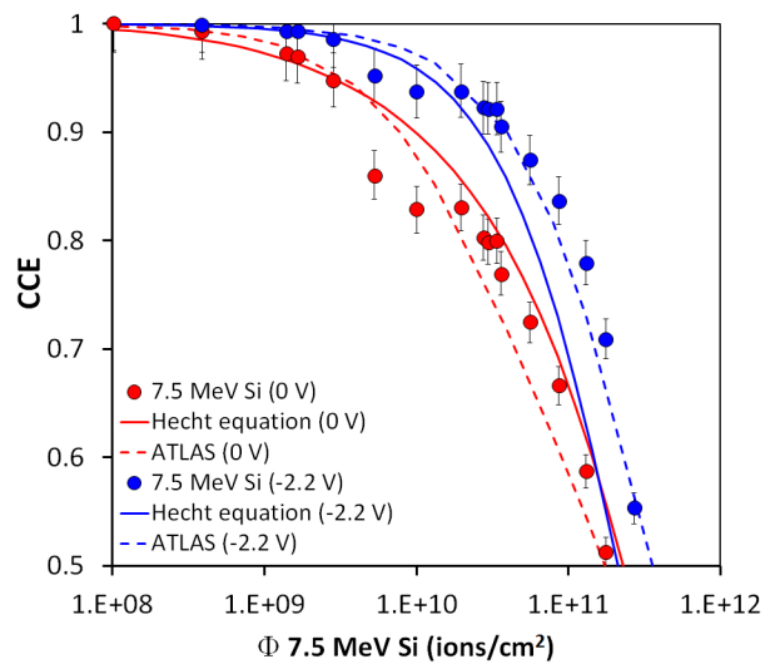

Fig. 8. CCE vs. 7.5 MeV Si fluence.

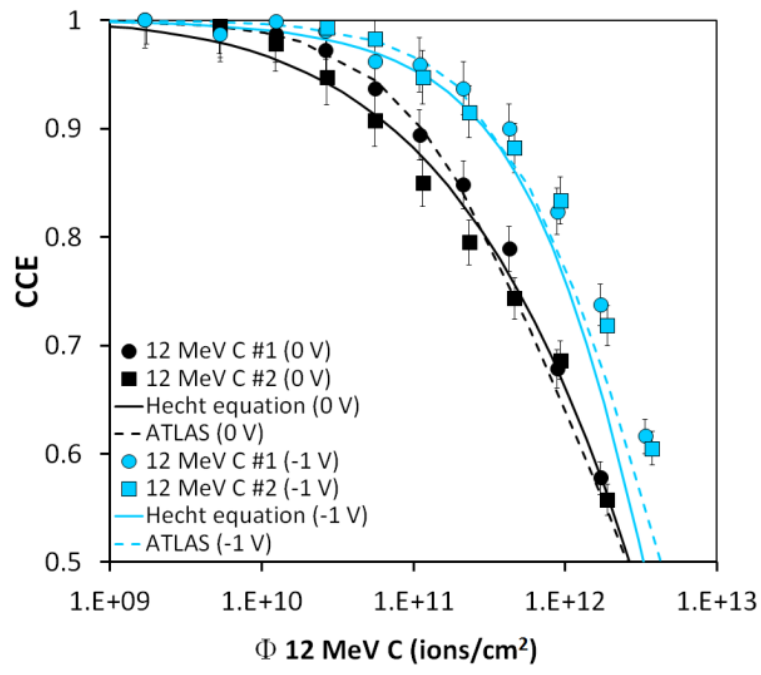

Fig. 9. CCE vs. $12 \mathrm{MeV}$ C fluence.

the expression $\tau_{e(h)}=\left(\sigma_{e(h)} v_{t h} N_{t}\right)^{-1}$. Hole and electron lifetimes were given equivalent values for each ATLAS simulation. It is assumed for this simulation that all defects will have midgap energy levels, that defects will have similar capture cross-sections for holes and electrons, and that the thermal velocity of both carriers is $7.7 \times 10^{6} \mathrm{~cm} / \mathrm{s}$ [15]. CCE values were calculated as the ratio between photocurrent simulated with a given set of carrier lifetimes to the initial photocurrent simulated with carrier lifetimes set to $10 \mathrm{~ns}$.

\subsection{Empirical determination of $k \Phi$}

The k-factors for $12 \mathrm{MeV} \mathrm{C}$ and 7.5 MeV Si ions were determined from CCE values measured in irradiated photodiodes and CCE values simulated with ATLAS. For each combination of ion species and energy, an approximate k-value was used with the Messenger-Spratt equation to convert lifetimes simulated with ATLAS to ion fluences. We make the approximation that $\mathrm{k}$-factors for holes and electrons are equal. A quadratic spline was fitted to ATLAS CCE values. The k-value for each ion was optimized by minimizing the nonlinear least squares fit between measured CCE values and CCE values interpolated from the quadratic spline. The k-factors calculated for biases between $+0.5 \mathrm{~V}$ and $-4 \mathrm{~V}$ were averaged to find $\mathrm{k}=1.29 \pm 0.36 \mathrm{~cm}^{2} / \mathrm{s}$ for $7.5 \mathrm{MeV}$ Si. For $12 \mathrm{MeV} \mathrm{C}$, $\mathrm{k}$ factors calculated for biases between $+0.5 \mathrm{~V}$ and $-1 \mathrm{~V}$ were averaged to find $\mathrm{k}=0.087 \pm 0.021 \mathrm{~cm}^{2} / \mathrm{s}$. Fig. $7 \mathrm{shows}$ the calculated Messenger-Spratt equation vs. fitted ATLAS lifetimes for measured C and Si fluences vs. $k \Phi$, the product of k-factor and fluence. These k-factors were used with the sum of the 1- and 2-carrier Hecht equations in (6) to calculate CCE as a function of $12 \mathrm{MeV}$ C fluence and 7.5 MeV Si fluence. CCE values in measured, calculated, and simulated data can be compared in Figs. 8 and 9 below.

\section{Results and discussion}

Fig. 8 compares CCE vs. fluence at biases of $0 \mathrm{~V}$ (partial depletion) and -2.2 V (full depletion) for a GaAs photodiode irradiated with 7.5 MeV Si. CCE values are measured in an ion-irradiated device, calculated with (6), and simulated with ATLAS. The error bars on measured data points correspond to the root sum square of uncertainties associated with photocurrent variation in a pristine photodiode, laser power stability, and currentvoltage measurements made with a semiconductor parameter analyzer. When the device was biased at $0 \mathrm{~V}$ as shown in the red datasets in Fig. 8, 69.2\% of $\mathrm{e}^{-} / \mathrm{h}$ pairs were generated in the depletion region and 30.8\% were generated in the field-free region. At a bias of $-2.2 \mathrm{~V}$ represented by the blue datasets in Fig. 8, 100\% of carriers were generated in the depletion region. It is clear that CCE degrades more quickly with fluence when the bias is set to $0 \mathrm{~V}$, so the device is less radiation tolerant when the intrinsic GaAs layer is partially depleted.

Fig. 9 shows measured, calculated, and simulated CCE values vs. fluence for GaAs photodiodes irradiated with $12 \mathrm{MeV} \mathrm{C}$. The photodiodes were partially depleted at biases of both $0 \mathrm{~V}$ and $-1 \mathrm{~V}$, but the depletion region expanded from $0.42 \mu \mathrm{m}$ to $0.54 \mu \mathrm{m}$ when bias was changed from $0 \mathrm{~V}$ to $-1 \mathrm{~V}$. At a bias of $-1 \mathrm{~V}, 86.6 \%$ of $\mathrm{e}^{-} / \mathrm{h}$ pairs were generated in the depletion region and $13.4 \%$ were generated in the field-free region. Even though the device is 


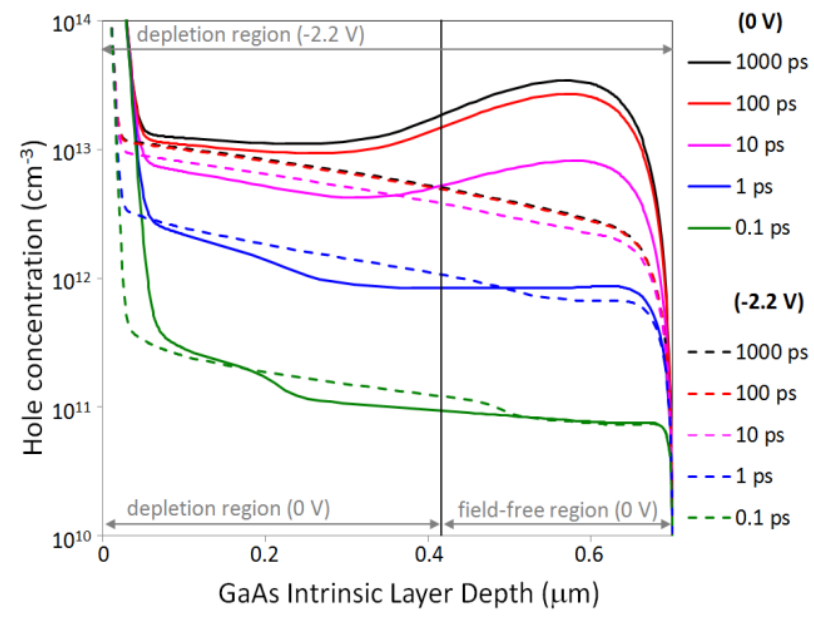

Fig. 10. Hole concentration vs. depth in the intrinsic layer for carrier lifetimes between $0.1 \mathrm{ps}$ and $1000 \mathrm{ps}$ at applied biases of $0 \mathrm{~V}$ or $-2.2 \mathrm{~V}$.

not fully depleted at a bias of $-1 \mathrm{~V}$, Fig. 9 shows that CCE degrades more slowly with fluence when a smaller percentage of holes must diffuse from the field-free region into the depletion region to contribute to CCE.

Fig. 10 shows ATLAS simulation results of hole concentration vs. depth for the intrinsic region of a GaAs diode biased at $0 \mathrm{~V}$ and $-2.2 \mathrm{~V}$. When the diode is fully biased at $-2.2 \mathrm{~V}$ (datasets shown with dotted lines), holes drift rapidly across the depletion region. When the diode is partially biased at $0 \mathrm{~V}$ (datasets shown with solid lines), the hole concentration shows a "bulge" in the field-free region between $0.9 \mu \mathrm{m}$ and $1.2 \mu \mathrm{m}$ when carrier lifetime is 10 ps or longer. Carrier lifetime is inversely proportional to displacement damage, so carrier lifetimes of 10 ps or greater correspond to lower levels of displacement damage while carrier lifetimes less than 10 ps indicate higher levels of displacement damage.

At low levels of displacement damage when few recombination sites have been generated, the hole concentration is elevated in the field-free region because holes can only escape by diffusing into the depletion region. As section 2.3 describes, holes generated in the field-free region can diffuse into the depletion region to the left, but an electric field at the interface between the GaAs intrinsic layer and the $n$-type $\mathrm{Al}_{0.25} \mathrm{Ga}_{0.75} \mathrm{As}$ (not shown) repels them from diffusing to the right. As displacement damage increases (and carrier lifetime decreases), holes recombine in the field-free region before they can diffuse to the depletion region and the hole concentration bulge disappears.

\section{Conclusions}

Measurements of CCE degradation as a function of both bias and fluence indicate that CCE degradation can be modeled as the sum of the 1-carrier and 2-carrier Hecht equation in partially depleted GaAs photodiodes. This degradation trend is consistent with the recombination of holes diffusing from the field-free region to the depletion region. These observations are supported by ATLAS simulations and by the calculation of CCE as a function of $k \Phi$ with the Hecht equation. In fully depleted photodiodes, CCE can be calculated with the 2-carrier Hecht equation. In partially depleted photodiodes, CCE can be calculated as the sum of the 2-carrier Hecht equation applied to electrons and holes generated in the depletion region and the 1-carrier Hecht equation applied to holes that diffuse from the field-free region into the depletion region. The physical understanding of CCE degradation mechanisms in partially depleted GaAs photodiodes can inform the design of radiation-hardened photodiodes.

\section{Acknowledgments}

The authors would like to thank Reinhard Brock for his expertise in translating [3] and Dan Buller for his assistance with the HVEC tandem accelerator. 
[1] J. R. M. Srour, C. J. Marshall, and P. W. Marshall, "Review of displacement damage effects in silicon devices," IEEE Transactions on Nuclear Science, vol. 50, pp. 653-670, June 2003.

[2] M. McPherson, B. K. Jones, and T. Sloan, "Effects of radiation damage in silicon p-i-n photodiodes," Semiconductor Science and Technology, vol. 12, pp. 1187-1194, 1997.

[3] K. Hecht, "Zum Mechanismus des lichtelektrischen primarstromes in isolierenden Kristallen," The European Physical Journal A, vol. 77, pp. 235-245, 1932.

[4] D. K. Schroder, "The concept of generation and recombination lifetimes in semiconductors," IEEE Transactions on Electron Devices, vol. ED-29, pp. 1336-1338, 1982.

[5] J. B. Gunn, "A general expression for electrostatic induction and its application to semiconductor devices," Solid-State Electronics, vol. 7, pp. 739-742, 1964.

[6] E. Vittone, Z. Pastuovic, M. B. H. Breese, J. Garcia Lopez, M. Jaksic, J. Raisanen, R. Siegele, A. Simon, G. Vizkelethy, harge collection efficiency degradation induced by $\mathrm{MeV}$ ions in semiconductor devices; model and experiment," Nuclear Instruments and Methods in Physics Research Section B: Beam Interactions with Materials and Atoms, vol. 372, pp. 128 - 142, 2016.

[7] G. C. Messenger and J. P. Spratt, "The effects of neutron irradiation on germanium and silicon," Proceedings of the IRE, pp. 1038-1044, June 1958.

[8] "ATLAS," 5.19.24.c ed: Silvaco, 2015, Device simulation software.

[9] E. L. S. Blansett, D. K. Serkland, M. J. Cich, K. M.Geib, G. M. Peake, R. M. Fleming, D. L. Wrobel, T. F. Wrobel, "Final report on LDRD project 105967: Exploring the increase in GaAs photodiode responsivity with increased neutron fluence," Sandia National Laboratories, Albuquerque, NM, 2008.

[10] E. S. Bielejec, G. Vizkelethy, N. R. Kolb, D. B. King, and B. L. Doyle, "Damage equivalence of heavy ions in silicon bipolar junction transistors," IEEE Transactions on Nuclear Science, vol. 53, pp. 3681 - 3686, 2006.

[11] J. F. Ziegler, M. D. Ziegler, and J. P. Biersack, "SRIM - The stopping and range of ions in matter," Nuclear Instruments and Methods in Physics Research Section B: Beam Interactions with Materials and Atoms, vol. 268, pp. 1818-1823, 2010.

[12] P. J. Griffin, J. G. Kelly, T. F. Luera, A. L. Barry, and M. S. Lazo, "Neutron damage equivalence in GaAs," Nuclear Science, IEEE Transactions on, vol. 38, pp. 1216-1224, 1991.

[13] J. S. Blakemore, "Semiconducting and other major properties of gallium arsenide," Journal of Applied Physics, vol. 53, pp. R123-R181, 1982.

[14] A. S. Grove, Physics and Technology of Semiconductor Devices. New York: John Wiley and Sons, Inc., 1967.

[15] P. A. Houston and A. G. R. Evans, "Saturation velocity of electrons in GaAs," IEEE Transactions on Electron Devices, pp. 584-586, June 1976.

[16] H. C. Casey Jr., D. D. Sell, and K. W. Wecht, "Concentration dependence of the absorption coefficient for nand p-type GaAs between 1.3 and $1.6 \mathrm{eV}$," Journal of Applied Physics, vol. 46, pp. 250-257, 1975.

[17] G.G. Arfken, H.J. Weber, Mathematical Methods for Physicists, Harcourt Academic Press, San Diego, San Francisco, New York, Boston, London, Sidney, Toronto, Tokyo, 2001.

\section{$\underline{\text { Appendix A }}$}

In this appendix, $\mathrm{CCE}$ is calculated using the Gunn theorem for a 1-D device in which photocurrent is excited by a charge generation profile $G(x)$ that is either 1) constant in space or 2) exponentially decaying in space. $G(x)$ is in units of $\mathrm{C} / \mathrm{m} / \mathrm{s}$. First, we will show that the Hecht equation for CCE can be derived from the Gunn theorem when a uniform charge distribution profile is assumed. Second, we will calculate CCE using the Gunn theorem with an exponential charge generation profile, which is more realistic for the experiment described in the full body of this paper. Finally, we will demonstrate that both CCE calculations agree with each other to within 3\% of each other for the CCE values measured in this experiment. For the sake of simplicity, only electrons will be considered; the treatment of holes is identical.

The induced current from a point charge according to the Gunn theorem is

$$
i(t)=-q \cdot v(x(t)) \cdot \frac{\delta E}{\delta V}(x(t)) .
$$


The Gunn function $\partial E / \partial V$ is non-zero in the depletion region, and in this region the velocity in (A.1) is the drift velocity. Equation (A.1) uses the values of the velocity and the Gunn field at the position $x$ where charge $q$ is at time $t$. The current calculated for a charge distribution of $q(t)=\rho(x, t) \cdot d x$ is

$$
i(t)=-\int \rho(x, t) \cdot v(x) \cdot \frac{\partial E}{\partial V}(x) \cdot d x
$$

Therefore, if the charge distribution in space and time is known, the current can be calculated. In case of the continuous photocurrent, the time differential of the current is zero after the initial transient:

$$
0=\frac{d i(t)}{d t}=-\int\left[\frac{d \rho}{d t} \cdot v \cdot \frac{\partial E}{\partial V}+\rho \cdot \frac{d v}{d t} \cdot \frac{\partial E}{\partial V}+\rho \cdot v \cdot \frac{d}{d t} \cdot \frac{\partial E}{\partial V}\right] \cdot d x
$$

This differential is zero for all times if the integrand is zero. The device used in this study is a diode. The Gunn field $\delta E / \delta V$ for a diode is the inverse of the depletion width and is therefore a constant, so the third term in the integrand is zero. Let's consider the remaining two terms.

The first term in (A.3) includes a term for charge distribution:

$$
\frac{d \rho}{d t}=\frac{\partial \rho}{\partial x} \cdot \frac{\partial x}{\partial t}+\frac{\partial \rho}{\partial t}=\frac{\partial \rho}{\partial x} \cdot v-\frac{\rho}{\tau}+G
$$

The charge distribution changes for two reasons. The charge is moving in space and time (first term) and the charge is changing locally due to recombination and generation. Recombination is defined with the term $-\rho / \tau$.

The second term in (A.3) includes a term for the derivative of velocity with respect to time:

$$
\frac{d v}{d t}=-\frac{\partial v}{\partial x} \cdot v
$$

Since the velocity field is constant in time, (A.5) shows that only the spatial dependence of the velocity field remains. It is negative because we are considering electrons only. The requirement that the integrand be zero becomes

$$
\frac{d \rho}{d x}+\frac{\rho}{\lambda} \cdot\left(1+\tau \cdot \frac{d v}{d x}\right)=\frac{\tau}{\lambda} \cdot G
$$

with $\lambda=\tau \cdot v$ drift length. Equation (A.6) is a first order differential equation where $v, \tau, G$ and $d v / d x$ are known functions of $x$.

The following assumptions are made to simplify the problem due to the specifics of our experiment to get an analytical form for the CCE.

- The lifetime is constant due to the approximately constant displacement damage.

- The electric field in the depletion region is very high and the drift velocity saturates. This eliminates the space differential of the velocity.

Using these assumptions, equation (A.6) becomes

$$
\frac{d \rho}{d x}+\frac{\rho}{\lambda}=\frac{\tau}{\lambda} \cdot G
$$

This equation has the solution [17] in the form of

$$
\rho(x)=e^{-\frac{x}{\lambda} \cdot \frac{\tau}{\lambda} \cdot \int_{0}^{x} e^{\frac{s}{\lambda}} \cdot G(s) \cdot d s .}
$$

\section{$\underline{\text { CCE with a uniform charge distribution }}$}

Consider a uniform generation profile, $G(s)=G_{0}$. Evaluating (A.8) gives a spatial charge profile of 


$$
\rho(x)=\tau \cdot G_{0} \cdot\left(1-e^{-\frac{x}{\lambda}}\right) .
$$

Substituting (A.9) into (A.2) and setting the depletion width to $x_{d}$ gives a current of

$$
i=-\lambda \cdot G_{0} \cdot\left(1-\frac{\lambda}{x_{d}} \cdot\left(1-e^{-\frac{x_{d}}{\lambda}}\right)\right) .
$$

In order to determine the CCE, current must be calculated for $\lambda \ll x_{d}$, that is, before displacement damage reduces the drift length $\lambda$ of electrons. The exponential term can be expanded in a Taylor series to achieve this. Expanding to the linear term results in zero current, but expanding to the quadratic term yields

$$
i(\lambda \rightarrow \infty)=-\frac{1}{2} \cdot G_{0} \cdot x_{d} .
$$

The CCE for a constant generation profile is

$$
C C E=\frac{i(\lambda)}{i(\lambda \rightarrow \infty)}=2 \cdot \frac{\lambda}{x_{d}} \cdot\left[1-\frac{\lambda}{x_{d}} \cdot\left(1-e^{-\frac{x_{d}}{\lambda}}\right)\right],
$$

which is the one carrier Hecht equation.

\section{CCE with an exponential charge distribution}

In the experiment, the charge generation profile is more realistically modeled as an exponential function than as a uniform distribution:

$$
G(x)=G_{0} \cdot e^{-A x}
$$

where $A$ is the absorption coefficient of the $808 \mathrm{~nm}$ light in GaAs. The value of $A$ in our case is $\sim 1.2 \mu \mathrm{m}^{-1}$, and $x$ can extend from 0 to $0.5 \mu \mathrm{m}$. The difference between the charge generation profile in the front and the back of the device is about $45 \%$. The next steps are to calculate the charge distribution, the current, and finally the CCE for the exponential generation profile. Substituting (A.13) into (A.8) gives the following charge generation profile:

$$
\rho(x)=\frac{G_{0} \cdot \tau}{1-A \cdot \lambda} \cdot\left(e^{-A x}-e^{-\frac{x}{\lambda}}\right)
$$

As a sanity check, (A.14) can be evaluated for $A=0$ to get equation (A.9) since an absorption coefficient of 0 equates to a uniform charge distribution. The current for the exponential generation distribution in (A.14) is

$$
i(\lambda)=-\frac{G_{0} \cdot \lambda}{x_{d}} \cdot\left[\frac{1}{A}+\frac{1}{1-A \cdot \lambda} \cdot\left(\lambda \cdot e^{-\frac{x_{d}}{\lambda}}-\frac{e^{-\frac{A x_{d}}{\lambda}}}{A}\right)\right] .
$$

It can be easily shown that equation (A.15) becomes equation (A.10) for $A \rightarrow 0$. The current for a very long drift length is

$$
i(\lambda \rightarrow \infty)=\frac{G_{0}}{x_{d} A^{2}} \cdot\left(1-A \cdot x_{d}-e^{-A x_{d}}\right) .
$$

437 The CCE for an exponential charge generation profile is 


\section{Convergence of CCE calculations using uniform and exponential charge generation profiles}

Although the calculation of CCE with an exponential charge generation profile given in (A.17) does not resemble the calculation of CCE with a uniform charge generation profile given in (A.12), the two equations converge as shown in Fig. A.1 below. Fig. A.1 demonstrates that the difference is very small between CCE values calculated with 1) a uniform charge generation in (A.12) (the Hecht equation) and 2) an exponential generation profile as in equation (A.17). Even for very low values of CCE (for which the drift length is $1 \mathrm{pm}$ ), the difference is less than $10 \%$. All measurements in the body of the paper resulted in CCE $>50 \%$. The figure below indicates that the difference between the exact formula and the Hecht equation is $<3 \%$. Based on the above calculations and the figure below, we consider that the calculation of CCE using the Hecht equation and a uniform charge generation profile is justified for the devices in this work.

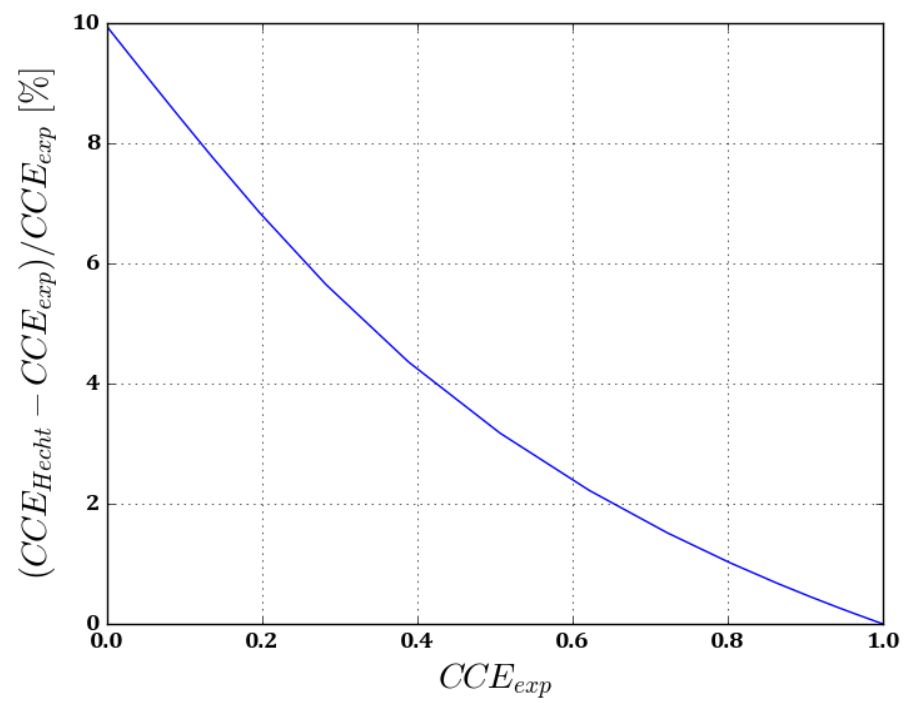

Fig. A.1: The difference between CCE calculated with a uniform charge generation profile (using the Hecht equation) and CCE calculated with an exponential charge generation profile, expressed as a percentage of CCE calculated with an exponential generation profile, vs. CCE calculated with an exponential generation profile.

44 